Anaesthesist 2021 · 70:888-894

https://doi.org/10.1007/s00101-021-00976-x

Angenommen: 26. April 2021

Online publiziert: 29. Juli 2021

(c) Springer Medizin Verlag $\mathrm{GmbH}$, ein Teil von Springer Nature 2021

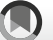

\section{Fokus allgemeine Intensivmedizin. Intensivmedizinische Studien aus 2020/2021}

\author{
M. Dietrich ${ }^{1} \cdot$ C. Beynon ${ }^{2} \cdot$ M. O. Fiedler $\cdot$ M. Bernhard ${ }^{3} \cdot$ P. Kümpers ${ }^{4} \cdot$ A. Hecker $^{5} \cdot$ \\ C. Jungk ${ }^{2} \cdot$ C. Nusshag ${ }^{6} \cdot$ D. Michalski ${ }^{7} \cdot$ T. Brenner $^{8} \cdot$ M. A. Weigand ${ }^{1} \cdot$ C. J. Reuß ${ }^{9}$ \\ ${ }^{1}$ Klinik für Anästhesiologie, Universitätsklinikum Heidelberg, Heidelberg, Deutschland \\ ${ }^{2}$ Neurochirurgische Klinik, Universitätsklinikum Heidelberg, Heidelberg, Deutschland \\ ${ }^{3}$ Zentrale Notaufnahme, Universitätsklinikum Düsseldorf, Heinrich-Heine-Universität, Düsseldorf, \\ Deutschland \\ ${ }^{4}$ Klinik für Allg. Innere Medizin und Notaufnahme sowie Nieren- und Hochdruckkrankheiten und \\ Rheumatologie, Universitätsklinikum Münster, Münster, Deutschland \\ ${ }^{5}$ Klinik für Allgemein-, Viszeral-, Thorax-, Transplantations- und Kinderchirurgie, Universitätsklinikum \\ Gießen und Marburg, Standort Gießen, Gießen, Deutschland \\ ${ }^{6}$ Klinik für Endokrinologie, Stoffwechsel und klinische Chemie/Sektion Nephrologie, Universitätsklinikum \\ Heidelberg, Heidelberg, Deutschland \\ ${ }^{7}$ Klinik und Poliklinik für Neurologie, Universitätsklinikum Leipzig, Leipzig, Deutschland \\ ${ }^{8}$ Klinik für Anästhesiologie und Intensivmedizin, Universitätsklinikum Essen, Essen, Deutschland \\ ${ }^{9}$ Klinik für Anästhesiologie und operative Intensivmedizin, Klinikum Stuttgart, Stuttgart, Deutschland
}

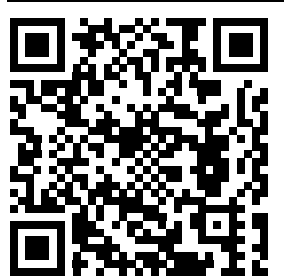

QR-Code scannen \& Beitrag online lesen

\section{Einleitung}

Mit dem Beginn der Coronapandemie im Frühjahr 2020 wurden die Gesundheitssysteme weltweit durch ein intensivmedizinisches Krankheitsbild an ihre Belastungsgrenzen gebracht. Obwohl das SARSCoV-2 nur bei einem kleinen Anteil der infizierten Patienten eine schwere Pneumonie mit Sepsis auslöst, kam es aufgrund der hohen Fallzahl zu einem massiven Bedarf an intensivmedizinischer Behandlungskapazität. Über 4 Mio. Menschen sind bisher (Stand: 08.07.2021) an einer COVID-19Sepsis verstorben [1]. Entsprechend fokussierte sich die intensivmedizinische Forschung im Jahr 2020/2021 auf das neuartige Krankheitsbild, und ein großer Anteil der erschienenen Original- und Übersichtsarbeiten betraf das SARS-CoV-2 bzw. das von ihm ausgelöste Krankheitsbild COVID19. Dennoch sind im vergangenen Jahr viele interessante Publikationen auch zu anderen intensivmedizinischen Fragestellungen erschienen. Wie in den vergangenen Jahren möchten wir im vorliegen- den Übersichtsartikel einige besonders relevante Veröffentlichungen vorstellen.

Da viele Studien unter Akronymen "gehandelt" werden, gibt $\bullet$ Tab. 1 einen Überblick über die im vergangenen Jahr erschienenen Studien unter gleichzeitiger

\section{Infobox 1}

Übersicht aller 5 Artikel zu den intensivmedizinischen Studien aus 2020/2021

- Fokus allgemeine Intensivmedizin: www. springermedizin.de/link/10.1007/s00101021-00976-x

- Fokus neurologische Intensivmedizin: www.springermedizin.de/link/10.1007/ s00101-021-00977-w

- Fokus neurochirurgische Intensivmedizin: www.springermedizin.de/link/10.1007/ s00101-021-00978-9

- Fokus Beatmung:www.springermedizin. de/link/10.1007/s00101-021-00979-8

- Fokus Nephrologie: www.springermedizin. de/link/10.1007/s00101-021-00980-1

Die Beiträge stehen Ihnen sofort nach der Fertigstellung auf www.springermedizin. de zur Verfügung. Bitte geben Sie dort den Beitragstitel in die Suche ein. 
Nennung der jeweiligen Akronyme (sofern vorhanden).

\section{Additive Therapie}

\section{Originalpublikationen}

Moskowitz A, Huang DT, Hou PC et al (2020) Effect of Ascorbic Acid, Corticosteroids, and Thiamine on Organ Injury in Septic Shock-The ACTS Randomized Clinical Trial. JAMA. https:// doi.org/10.1001/jama.2020.11946

Hwang SY, Ryoo SM, Park JE et al (2020)

Combination therapy of vitamin $\mathrm{C}$ and thiamine for septic shock: a multi-centre, double-blinded randomized, controlled study. Intensive Care Med. https://doi.org/10.1007/ s00134-020-06191-3

Iglesias J, Vassallo AV, Patel VV et al (2020) Outcomes of Metabolic Resuscitation Using Ascorbic Acid, Thiamine, and Glucocorticoids in the Early Treatment of Sepsis-The ORANGES Trial. CHEST. https://doi.org/10.1016/j.chest. 2020.02.049

Sevransky JE, Rothman RE, Hager DN et al (2021) Effect of Vitamin C, Thiamine, and Hydrocortisone on Ventilator and VasopressorFree Days in Patients With Sepsis-The VICTAS Randomized Clinical Trial. JAMA. 325(8):742-751. https://doi.org/10.1001/jama. 2020.24505

In einer im Jahr 2017 von Paul Marik publizierten retrospektiven Vorher-nachher-Analyse ergaben sich Hinweise für ein deutlich verbessertes Überleben von Patienten mit septischem Schock durch die hochdosierte Gabe von Vitamin C, Thiamin und Hydrocortison (HAT-Therapie). Bei einer Patientenkohorte vor Einführung der Therapie lag die Sterblichkeit bei ca. $40 \%$, nach Einführung der additiven Gabe aus 1,5 g Vitamin C 6-stündlich, Thiamin $200 \mathrm{mg}$ 12-stündlich, jeweils mit einer Therapiedauer von 4 Tagen und $50 \mathrm{mg}$ Hydrocortison 6-stündlich für 7 Tage reduzierte sich die Sterblichkeit unter $10 \%$. Zusätzlich zeigte die mit der HAT-Therapie behandelte Kohorte einen deutlich rascheren Abfall des SOFAScores [2]. Als Folge dieser Ergebnisse wurden mehrere prospektive, randomisierte kontrollierte Studien aufgelegt, um die HAT-Therapie zu untersuchen. Bereits 2019/2020 wurden erste Studien mit gemischten Ergebnissen veröffentlicht: Die CITRIS-ALI-Studie zeigte eine Verbesserung des Überlebens durch die Gabe von hochdosiertem Vitamin C ( $50 \mathrm{mg} / \mathrm{kgKG})$ 6-stündlich bei Patienten mit Sepsis und
ARDS, wohingegen die VITAMINS-Studie keinen Einfluss des HAT-Bündels auf das Überleben zeigte [3, 4]. Nebenwirkungen des hochdosierten Vitamin $C$ und Thiamins wurden in beiden Studien nicht beobachtet. Jedoch gibt es Fallberichte über Nierenversagen nach hochdosierter Gabe von Vitamin C $[5,6]$. Außerdem wird vor der Gabe von Vitamin $C$ bei Patienten mit Glucose-6-phosphatdehydrogenase-Mangel aufgrund des Risikos für hämolytische Krisen gewarnt.

Auch die 2020 in JAMA veröffentlichte ACTS-Studie untersuchte placebokontrolliert das klassische HAT-Bündel entsprechend der Studie von Marik et al. [2, 7]. Die Voraussetzungen für den Einschluss in die Studie waren eine vermutete oder bestätigte Infektion und Vasopressorbedarf. Ein erhöhtes Lactat gemäß der Sepsis-3-Definition für einen septischen Schock wurde nicht gefordert. Die Ausschlusskriterien umfassten einen bekannten Glucose-6phosphatdehydrogenase-Mangel, schwere Nierenfunktionseinschränkungen sowie ein Nierensteinleiden. Interventionsgruppe $(n=101)$ und Kontrollgruppe $(n=99)$ umfassten insgesamt 200 Patienten und zeigten bei Einschluss keine Unterschiede. Häufigster Sepsisfokus in beiden Gruppen war die Lunge (ca. $30 \%$ ), weitere häufige Ursache der Sepsis waren intraabdominelle Infektionen (Interventionsgruppe 32\% bzw. Kontrollgruppe $25 \%$ ). Primärer Endpunkt war die Kinetik des SOFA-Scores innerhalb von $72 \mathrm{~h}$ nach Studieneinschluss. Der initiale SOFA-Score lag in beiden Gruppen bei 9 Punkten. In der Gruppe, die die HAT-Therapie erhielt, fiel er innerhalb von $72 \mathrm{~h}$ auf 4,4 Punkte, in der Kontrollgruppe auf 5,1 Punkte. Somit ergab sich kein Unterschied. Auch die Sterblichkeit nach 30 Tagen unterschied sich nicht zwischen den Gruppen (34,7\% vs. 29,3\%). Jedoch zeigten sich bei der mit der HAT-Therapie behandelten Gruppe signifikant mehr schockfreie Tage innerhalb der ersten 7 Tage (5 vs. 4 Tage) [7].

Die in Südkorea durchgeführte ATESSStudie untersuchte ebenfalls die Gabe von Vitamin $C$ und Thiamin bei Patienten mit septischem Schock [8]. Jedoch unterschied sich die Studie im Design von der ACTSStudie bezüglich der Gabe von Hydrocortison und der Vitamin-C-Dosierung. In beiden Gruppen erhielten Patienten mit einem Noradrenalinbedarf von mehr als $0,2 \mu \mathrm{g} / \mathrm{kgKG}$ und min zusätzlich Hydrocortison und Vasopressin. Vitamin C wurde 12stündlich mit einer Dosierung von $50 \mathrm{mg} /$ kgKG mit einer maximalen Einzeldosis von $3 \mathrm{~g}$ verabreicht. Es wurden erwachsene $\mathrm{Pa}$ tienten mit septischem Schock (gemäß Sepsis-3-Definition) in der Notaufnahme eingeschlossen; auch in dieser Studie waren Nierensteine ein Ausschlusskriterium. Insgesamt wurden 111 Patienten untersucht: 53 in der Interventions- und $58 \mathrm{~Pa}$ tienten in der Placebogruppe ohne signifikante Unterschiede zwischen den Vergleichsgruppen. Der häufigste Infektionsfokus war in beiden Gruppen das Abdomen. Die Hälfte der Patienten hatte bei Einschluss einen Vitamin-C-Mangel, während nur ca. jeder Zehnte einen Thiaminmangel aufwies. Die Veränderung des SOFAScores innehrhalb von $72 \mathrm{~h}$ war auch in dieser Analyse der primäre Endpunkt. Dieser lag bei Einschluss in beiden Gruppen bei 8 Punkten. In beiden Gruppen nahm der SOFA-Score innerhalb von $72 \mathrm{~h}$ um 3 Punkte ab. Sowohl die Sterblichkeit als auch alle weiteren sekundären Endpunkte und Subgruppenanalysen bezüglich des primären Endpunkts unterschieden sich nicht zwischen den Gruppen.

Die ebenfalls 2020 publizierte ORANGES-Studie untersuchte placebokontrolliert den Einfluss des HAT-Bündels auf die Veränderung des SOFA-Scores und die Dauer der Vasopressortherapie bei Patienten mit Sepsis und septischem Schock [9]. Auch in dieser Studie wurden Patienten mit Glucose-6-phosphatdehydrogenaseMangel ausgeschlossen. Die HAT-Gruppe (68 Patienten) und die Kontrollgruppe (69 Patienten) zeigten bei Einschluss keine signifikanten Unterschiede. Häufigster Infektionsfokus war mit einem Anteil von $43 \%$ die Lunge, und $31 \%$ der Patienten hatten eine Harnwegsinfektion. Der initiale SOFA-Score lag im Mittel bei 8 Punkten. Die Hälfte der Patienten hatte bei Einschluss einen Vitamin-C-Mangel, ca. 10\% einen schweren Mangel. Die mit der HATTherapie behandelten Patienten erholten sich nach im Mittel $27 \mathrm{~h}$ schneller von dem Schockzustand, verglichen mit der Kontrollgruppe mit einer Dauer der Vasopressortherapie von $53 \mathrm{~h}$. Bezüglich der Abnahme des SOFA-Scores innerhalb der ersten $72 \mathrm{~h}$ und der Sterblichkeit zeigten 
Tab. 1 Intensivmedizinische Studien 2020/21 und die Bedeutung der Akronyme

\begin{tabular}{|c|c|c|c|}
\hline Akronym & Bedeutung des Akronyms & Originaltitel der Studie & Ergebnis - Kurzzusammenfassung \\
\hline ACTS & $\begin{array}{l}\text { Ascorbic Acid, Corticoste- } \\
\text { roids, and Thiamine in } \\
\text { Septic Shock }\end{array}$ & $\begin{array}{l}\text { Effect of Ascorbic Acid, Corticosteroids, and } \\
\text { Thiamine on Organ Injury in Septic Shock - The } \\
\text { ACTS Randomized Clinical Trial }\end{array}$ & $\begin{array}{l}\text { Bei Patienten mit Sepsis zeigte die Gabe von Vita- } \\
\text { min C, Thiamin und Hydrocortison keinen Einfluss auf } \\
\text { die Kinetik des SOFA-Scores innerhalb von } 72 \mathrm{~h} \text { und } \\
\text { die 30-Tage-Sterblichkeit }\end{array}$ \\
\hline ATESS & $\begin{array}{l}\text { Ascorbic Acid and Thiami- } \\
\text { ne Effect in Septic Shock }\end{array}$ & $\begin{array}{l}\text { Combination therapy of vitamin C and thiamine } \\
\text { for septic shock: a multi-centre, double-blinded } \\
\text { randomized, controlled study }\end{array}$ & $\begin{array}{l}\text { Bei Patienten mit septischem Schock zeigte die Gabe } \\
\text { von Vitamin C und Thiamin keinen Einfluss auf die } \\
\text { Kinetik des SOFA-Scores innerhalb von } 72 \mathrm{~h}\end{array}$ \\
\hline ORANGES & $\begin{array}{l}\text { Outcomes of Metabolic } \\
\text { Resuscitation Using Ascor- } \\
\text { bic Acid, Thiamine, and } \\
\text { Glucocorticoids in the Early } \\
\text { Treatment of Sepsis }\end{array}$ & $\begin{array}{l}\text { Outcomes of Metabolic Resuscitation Using } \\
\text { Ascorbic Acid, Thiamine, and Glucocorticoids in } \\
\text { the Early Treatment of Sepsis - The ORANGES } \\
\text { Trial }\end{array}$ & $\begin{array}{l}\text { Die Therapiekombination aus Vitamin C, Thiamin und } \\
\text { Hydrocortison hatte keinen Einfluss auf die Abnahme } \\
\text { des SOFA-Scores innerhalb von } 72 \mathrm{~h} \text { und die Sterb- } \\
\text { lichkeit, führte jedoch zu einer schnelleren Reduktion } \\
\text { des Vasopressorbedarfs }\end{array}$ \\
\hline VICTAS & $\begin{array}{l}\text { Vitamin C, Thiamine, and } \\
\text { Steroids in Sepsis }\end{array}$ & $\begin{array}{l}\text { Effect of Vitamin C, Thiamine, and Hydrocortiso- } \\
\text { ne on Ventilator and Vasopressor-Free Days in } \\
\text { Patients With Sepsis - The VICTAS Randomized } \\
\text { Clinical Trial }\end{array}$ & $\begin{array}{l}\text { Die Therapiekombination aus Vitamin C, Thiamin } \\
\text { und Hydrocortison führte zu keinem signifikanten } \\
\text { Unterschied bezüglich der beatmungs- und vaso- } \\
\text { pressorfreien Tage }\end{array}$ \\
\hline CITRIS-ALI & $\begin{array}{l}\text { (Vitamin) C Infusion for } \\
\text { Treatment in Sepsis In- } \\
\text { duced Acute Lung Injury }\end{array}$ & $\begin{array}{l}\text { Effect of Vitamin C Infusion on Organ Failure } \\
\text { and Biomarkers of Inflammation and Vascular } \\
\text { Injury in Patients with Sepsis and Severe Acute } \\
\text { Respiratory Failure - The CITRIS-ALI Random- } \\
\text { ized Clinical Trial }\end{array}$ & $\begin{array}{l}\text { Bei Patienten mit Sepsis und ARDS führte die Gabe } \\
\text { von Vitamin C nicht zu einer Veränderung der Kine- } \\
\text { tik von SOFA-Score, C-reaktivem Protein- und des } \\
\text { Thrombomodulinspiegels. Patienten, die Vitamin-C } \\
\text { erhielten, hatten eine geringere Sterblichkeit }\end{array}$ \\
\hline VITAMINS & $\begin{array}{l}\text { Vitamin C, Hydrocortisone } \\
\text { and Thiamine in Patients } \\
\text { with Septic Shock }\end{array}$ & $\begin{array}{l}\text { Effect of Vitamin C, Hydrocortisone, and Thiami- } \\
\text { ne vs. Hydrocortisone Alone on Time Alive and } \\
\text { Free of Vasopressor Support Among Patients } \\
\text { with Septic Shock - The VITAMINS Randomized } \\
\text { Clinical Trial }\end{array}$ & $\begin{array}{l}\text { Die hochdosierte Gabe von Vitamin C und Thiamin } \\
\text { hatte keinen Einfluss auf das Überleben und den } \\
\text { Vasopressorbedarf, verglichen mit Hydrocortison } \\
\text { alleine }\end{array}$ \\
\hline HALT-IT & $\begin{array}{l}\text { Haemorrhage Alleviation } \\
\text { With Tranexamic Acid-In- } \\
\text { testinal System }\end{array}$ & $\begin{array}{l}\text { Effects of a high-dose } 24-h \text { infusion of tranexa- } \\
\text { mic acid on death and thromboembolic events } \\
\text { in patients with acute gastrointestinal bleed- } \\
\text { ing (HALT-IT): an international randomised, } \\
\text { double-blind, placebo-controlled trial }\end{array}$ & $\begin{array}{l}\text { Tranexamsäure verringerte innerhalb der ersten } \\
5 \text { Tage nach oberer oder unterer gastrointestinaler } \\
\text { Blutung nicht die Sterblichkeit aufgrund eines Blu- } \\
\text { tungsereignisses }\end{array}$ \\
\hline DESIRE & $\begin{array}{l}\text { Dexmedetomidine for } \\
\text { Sepsis in ICU Randomized } \\
\text { Evaluation }\end{array}$ & $\begin{array}{l}\text { Effect of Dexmedetomidine on Mortality and } \\
\text { Ventilator-Free Days in Patients Requiring Me- } \\
\text { chanical Ventilation With Sepsis: A Randomized } \\
\text { Clinical Trial }\end{array}$ & $\begin{array}{l}\text { Eine Sedierung mit Dexmedetomidin führte nicht zu } \\
\text { einem Unterschied bezüglich der 28-Tage-Sterblich- } \\
\text { keit oder der beatmungsfreien Tage, verglichen mit } \\
\text { einer Sedierung ohne Dexmedetomidin }\end{array}$ \\
\hline $\begin{array}{l}\text { NOSTRA- } \\
\text { DAMUS }\end{array}$ & $\begin{array}{l}\text { Identification of novel } \\
\text { sublingual parameters } \\
\text { to analyze and diagnose } \\
\text { microvascular dysfunction } \\
\text { in sepsis }\end{array}$ & $\begin{array}{l}\text { Identification of novel sublingual parameters to } \\
\text { analyze and diagnose microvascular dysfuncti- } \\
\text { on in sepsis: the NOSTRADAMUS study }\end{array}$ & $\begin{array}{l}\text { Der mittels sublingualer Videomikroskopie gemesse- } \\
\text { ne Microvascular Health Score erscheint anwendbar } \\
\text { als ein neuer Parameter zur standardisierten Ein- } \\
\text { schätzung der mikrozirkulatorischen Dysfunktion bei } \\
\text { Patienten mit Sepsis }\end{array}$ \\
\hline
\end{tabular}

sich keine Unterschiede zwischen den Gruppen. Die Sterblichkeit war, verglichen mit der ATESS- und der ACTS-Studiemit unter $20 \%$ in der ORANGES-Studie deutlich geringer. Renale Komplikationen traten in beiden Studiengruppen in gleichem Maße auf [9].

Die Ergebnisse der vorzeitig aufgrund administrativer Gründe abgebrochenen VICTAS-Studie wurden im Februar 2021 in JAMA veröffentlicht [10]. Für die Studie wurden 501 Patienten mit respiratorischem oder kardiozirkulatorischem Versagen im Rahmen einer Sepsis in 43 Krankenhäusern in den Vereinigten Staaten eingeschlossen. Die Interventionsgruppe der randomisierten kontrollierten Studie erhielt 6-stündlich 1,5 g Vitamin C, $100 \mathrm{mg}$ Thiamin und $50 \mathrm{mg}$ Hydrocortison mit einer Therapiedauer von 4 Tagen. Zusätzlich konnten in beiden Gruppen durch die behandelnden Ärzte Hydrocortison verabreicht werden; die Studienmedikation wurde dann entsprechend angepasst, um eine zu hohe Dosierung zu vermeiden. Der primäre Endpunkt war kombiniert aus den Tagen ohne Beatmung oder Katecholamintherapie bis Tag 30 nach Studieneinschluss. Beide Gruppen hatten bei Einschluss einen SOFA-Score von 9 Punkten, und häufigster Infektionsfokus war mit 37,1\% bzw. 39,7\% die Lunge. Die Ergebnisse zeigten keinen Unterschied bezüglich des primären Endpunkts aus beatmungs- und vasopressorfreien Tagen zwischen Interventions- (25 Tage) und Kontrollgruppe (26 Tage). Auch die Analyse der Sterblichkeit nach 30 und 180 Tagen sowie die Auswertung weiterer sekundärer Endpunkte ergaben keine Unterschiede. Die Aussagekraft der Ergebnisse der VICTAS-Studie werden durch den vorzeitigen Studienabbruch eingeschränkt; ursprünglich war der Einschluss von 2000 Patienten geplant [10].

In der Zusammenschau zeigte keine der vorgestellten Studien einen Überlebensvorteil durch die hochdosierte Gabe von Vitamin C und Thiamin in Kombination mit Hydrocortison. Auch die in der Studie von Marik et al. gezeigte schnellere Erholung 
der Organfunktion konnte in keiner der Studien reproduziert werden [2]. Sowohl in der ACTS- als auch in der ORANGES-Studie zeigte sich eine schnellere hämodynamische Stabilisierung bei Patienten, die die HAT-Therapie erhalten hatten, jedoch ohne Einfluss auf die Organdysfunktion oder das Gesamtüberleben [7, 9].

Weitere Analysen sind notwendig, um herauszufinden, welche Patienten, bezogen auf Merkmale wie Infektionsfokus, Katecholaminbedarf, Vorliegen von Mikrozirkulationsstörungen, Ausmaß des Organversagens, Alter und Geschlecht, von der HAT-Therapie profitieren, um eine individualisierte Sepsistherapie zu ermöglichen und die schnellere Schockerholung möglicherweise in ein verbessertes Überleben umsetzen zu können. Die deutliche Reduktion der Sterblichkeit und des Organversagens bei Patienten mit Sepsis in der retrospektiven Analyse von Marik et al. konnte bisher nicht konsistent in prospektiven Studien reproduziert werden [2].

\section{Hämodynamische Therapie und Mikrozirkulation}

\section{Originalpublikation}

Hasegawa D, Sato R, Prasitlumkum $\mathrm{N}$ et al (2021) Effect of ultra-short-acting $\beta$-blockers on mortality in patients with persistent tachycardia despite initial resuscitation: a systematic review and meta-analysis of randomized controlled trials. CHEST. https:// doi.org/10.1016/j.chest.2021.01.009

Eine Tachykardie in der Sepsis führt zu vermehrtem kardialen Sauerstoffverbrauch und ist mit einer höheren Sterblichkeit assoziiert. Eine medikamentöse Frequenzkontrolle kann auch bei hämodynamisch instabilen Patienten zu einer Verbesserung der Hämodynamik führen.

In einer Metaanalyse von 7 randomisierten kontrollierten Studien wurde als primärer Endpunkt der Effekt von den kurz wirksamen $\beta$-Blockern Esmolol und Landiolol bei septischen Patienten zur Therapie einer persistierenden Tachykardie nach initialer Stabilisierung durch Flüssigkeitsgabe untersucht [11]. Primärer Endpunkt war die Sterblichkeit nach 28 Tagen. In 6 von 7 eingeschlossenen Studien wurde Esmolol verwendet, nur in einer Landiolol. In den meisten der Studien hatten die Pa- tienten bei Einschluss einen ausgeprägten Katecholaminbedarf. Es wurden insgesamt 5 Studien aus China, eine aus Japan und eine Studie aus Italien in die Analyse einbezogen. Nur 6 dieser Studien untersuchten die 28-Tage-Sterblichkeit, sodass $572 \mathrm{~Pa}$ tienten in die Analyse des primären Endpunkts eingeschlossen werden konnten. Die Gabe der kurz wirksamen $\beta$-Blocker erfolgte mittels Perfusor, mit Dosisanpassung um eine Normalisierung der Herzfrequenz zu erreichen. In 5 Studien war eine Tachykardie definiert als Herzfrequenz über 95 bzw. 100 Schläge/min. Es zeigte sich nicht nur eine verminderte Herzfrequenz, sondern auch eine verminderte 28-Tage-Sterblichkeit mit einem relativen Risiko (RR) von 0,68 (95\%-KI: 0,58-0,85) bei Patienten, die einen kurz wirksamen $\beta$-Blocker erhalten hatten. Zusätzlich zeigte sich ein geringeres Lactat $72 \mathrm{~h}$ nach Einschluss in der Interventionsgruppe. Bezüglich des mittleren arteriellen Blutdrucks, des Herzzeitvolumens und des Noradrenalinbedarfs zeigten sich keine Unterschiede durch die $\beta$-Blocker-Therapie. Zusammenfassend gibt es Hinweise darauf, dass die Gabe eines kurz wirksamen $\beta$-Blockers zur Therapie einer persistierenden Tachykardie nach initialer Stabilisierung einen günstigen Effekt auf das Überleben von septischen Patienten haben könnte. Negative Effekte auf die hämodynamische Stabilität konnten durch diese Metaanalyse nicht gezeigt werden, sodass speziell die Gabe von sehr kurz wirksamen $\beta$-Blockern trotz bestehendem Vasopressorbedarf bei nach Wirkung titrierter Gabe sicher erscheint. Jedoch wurden bei den meisten der in dieser Metaanalyse eingeschlossenen Studien Patienten mit eingeschränkter kardialer Funktion ausgeschlossen. Somit ist unklar, ob diese Patientengruppe ebenfalls von der $\beta$-Blockade profitieren würde.

\section{Originalpublikation}

Brechot N, Hajage D, Kimmoun A et al (2020) Venoarterial extracorporeal membrane oxygenation to rescue sepsis-induced cardiogenic shock: a retrospective, multicentre, international cohort study. Lancet. https://doi. org/10.1016/S0140-6736(20)30733-9

Das Krankheitsbild des septischen Schocks hat trotz intensivmedizinischer Therapie eine extrem hohe Sterblichkeit [12, 13]. Eine besondere Herausforderung stellen
Patienten mit ausgeprägter kardiogener Komponente des septischen Schocks dar. Diese Patienten zeigen ein Mischbild aus distributivem Schock mit Vasoplegie und Volumenmangel sowie einem kardiogenen Schock, induziert durch eine septische Kardiomyopathie. Bisher war die einzige Möglichkeit, diese Patienten durch eine differenzierte Katecholamin- und Volumentherapie bestmöglich zu unterstützen.

Eine von Brechot et al. publizierte retrospektive Analyse untersuchte den Effekt einer venoarteriellen (va-)ECMO-Therapie als Rescue-Strategie bei Patienten mit septischem Schock, verursacht durch eine ausgeprägte septische Kardiomyopathie [14]. Durch die va-ECMO wird desoxygeniertes Blut aus der V. cava abgesaugt, extrakorporal oxygeniert und über eine arterielle Kanüle meist in der Leiste zurückgeführt, um die Organperfusion retrograd zu unterstützen. Die Daten der Studie wurden zwischen 2008 und 2018 an 5 universitären ECMO-Zentren erhoben. Eingeschlossen wurden erwachsene Patienten mit septischem Schock und einem Herzindex $\leq 3 \mathrm{~m}^{2} \mathrm{KOF}$ oder einer linksventrikulären Ejektionsfraktion $\leq 35 \%$ in Kombination mit einem „vasoactive-inotropic score $^{\prime \prime} \geq 75 \mu \mathrm{g} / \mathrm{kgKG} \cdot$ min oder einem Lactat von $\geq 4 \mathrm{mmol} / \mathrm{l}$. Der Vasoactive-inotropic score gewichtet verschiedene Katecholamine mit einem Faktor und vereint diese zu einem Score, um sie vergleichbar zu machen. Insgesamt wurden 82 Patienten, die eine va-ECMO-Therapie erhalten hatten, mit 130 konventionell behandelten Patienten verglichen. Primärer Endpunkt der Analyse war das Überleben nach 90 Tagen. Bei Einschluss zeigten sich deutliche Unterschiede zwischen beiden Gruppen: Die mittels ECMO therapierten Patienten waren deutlich jünger, Fokus der Infektion war häufiger die Lunge, und sie zeigten eine ausgeprägtere hämodynamische Instabilität sowie einen höheren SOFA-Score. Deswegen wurde zusätzlich eine gewichtete Analyse der Gruppen durchgeführt.

Die Ergebnisse der Analyse zeigten ein besseres 90-Tage-Überleben derva-ECMOGruppe im Vergleich zur konventionellen Therapie ( $60 \%$ vs. $25 \%$ ). Zusätzlich zeigte sich eine deutliche schnellere Reduktion des Lactats als Zeichen der Wiederherstellung der Gewebeperfusion/-oxygenierung und des Katecholaminbedarfs. Die 
Ergebnisse zeigen, dass die Implantation einer va-ECMO eine sinnvolle Rescue-Therapie bei Patienten mit septischem Schock mit ausgeprägter kardiogener Komponente sein kann. Vor allem bei Patienten, bei denen durch eine konventionelle Therapie keine adäquate Gewebe- und Organperfusion erreicht werden kann, sollte an diese Therapieoption gedacht werden, um die akute Phase des Schocks bis zur Erholung der kardialen Funktion zu überbrücken.

\section{Originalpublikation}

Rovas A, Sackarnd J, Rossaint J et al (2021) Identification of novel sublingual parameters to analyze and diagnose microvascular dysfunction in sepsis: the NOSTRADAMUS study. Crit Care. 25(1):112. https://doi.org/10. 1186/s13054-021-03520-w

In den letzten 15 Jahren hat die ständige Verbesserung der sublingualen $\mathrm{Mi}$ krozirkulationsmessung bei (Intensiv-)Patienten erheblich zum Verständnis der Pathophysiologie beim Schock beigetragen. Trotz ständiger Verbesserungen der Kameratechnik lassen sich so aber bislang nur schwere Störungen der Mikrozirkulation semiquantitativ abschätzen („eyeballing $\left.{ }^{\prime}\right)$. - sprich: Die Sensitivität der Methode ist nicht besonders gut.

Genau an dieser Stelle setzt die Studie von Rovas et al. an. Mithilfe des Glycocheck $^{\mathrm{TM}}$-Systems (GlycoCheck B.V., Maastricht, Niederlande) gelang es den Autoren, in Kooperation mit Wissenschaftlern der Fa. Microvascular Health Solutions, die Fließeigenschaften der Erythrozyten viel genauer als bisher - und v. a. vollautomatisch - zu quantifizieren. Vereinfacht gesagt, misst die Glycocheck-Software, wie schnell die Erythrozyten fließen, und wie stark sie dabei von einer idealen „Fahrrinne" zur Seite hin abweichen. Je stärker die Abweichung nach außen, hin zur Gefäßwand, ist, desto größer wird die „perfused boundary region" (PBR), ein inverser Schätzwert für die Dicke der (protektiven) endothelialen Glykokalyx (vereinfacht hohePBR = "dünne" Glykokalyx). DerClou der Software ist aber, dass über den Durchmesser der Erythrozyten-Fahrrinne jeder kleinste Gefäßabschnitt einem ganz konkreten Gefäßdurchmesser zwischen 4 und $25 \mu \mathrm{m}$ zugeordnet werden kann (in 1- $\mu \mathrm{m}$ Klassen).
Anhand der Messwerte von insgesamt 17 gesunden Probanden und 34 Sepsispatienten konnten die Autoren zeigen, dass die Gefäßdichte der Sepsispatienten ausschließlich in den Durchmesserklassen 5, 6 und $7 \mu \mathrm{m}$ reduziert war $(-63 \%,-42 \%$ und-28\% im Vergleich zu den Kontrollen), während die übrigen Durchmesserklassen von 8 bis $25 \mu \mathrm{m}$ sich nicht zwischen den Gruppen unterschieden. Die Dichte dieser kleinsten Kapillaren ( $\leq 7 \mu$ m Durchmesser) zeigte zudem robuste inverse Korrelationen mit Interleukin-6, Prokalzitonin, Lactat und dem SOFA-Score, während diese in größeren Gefäßen fehlten oder sogar teilweise umgekehrt waren. Anschließend setzten die Autoren die jeweilige Erythrozyten-Geschwindigkeit (VRBC) von Kapillaren $(\leq 7 \mu \mathrm{m})$ mit denen zuführender Gefäße ( $\geq 10 \mu \mathrm{m}$, „feeding vessels“) in Beziehung. Dabei zeigte sich, dass gesunde Individuen eine relativ konstante mediane kapilläre VRBC zu haben scheinen, und zwar unabhängig von der zugehörigen VRBC in den zuführenden Gefäßen. Die Autoren spekulieren, dass die Einhaltung eines engen VRBC-Bereichs für ein optimales Angebot-Nachfrage-Verhältnis in den Kapillaren wichtig sein könnte. In der Sepsis entspricht die kapilläre VRBC hingegen 1:1 der VRBC in den Feeding vessels. Dieser Befund spricht dafür, dass die Anzahl der durchbluteten Kapillaren bei Sepsispatienten fixiert ist und unempfindlich gegenüber lokalen Variationen des metabolischen Bedarfs im Gewebe zu sein scheint. Basierend auf diesem Befund berechneten die Autoren in beiden Gruppen einen völlig neuen Parameter: die Kapillar-Rekrutierung (CR, in \%). Die CR betrug bei Gesunden im Mittel $80 \%$ während Sepsispatienten bei nur $10 \%$ lagen. Abschließend wurden die verschiedenen neuen Variablen (Kapillardichte, CR und PBR) zu einem Microvascular Health Score (MVHS) fusioniert. Dieser MVHS nahm mit zunehmender Sepsisschwere deutlich ab und korrelierte gut mit zahlreichen Inflammationsparametern.

Inwieweit diese neue Technik bzw. der MVHS tatsächlich relevante Endpunkte bei Sepsis-Patienten vorhersagen kann, bleibt abzuwarten. Die Autoren haben aber auch schon zwei prospektive, longitudinale $\mathrm{Ob}$ servationsstudien in der Notaufnahme und auf der Intensivstation gestartet. Gut vor- stellbar, dass der MVHS also den bisherigen Goldstandard (Eyeballing über alle Gefäße $<20 \mu \mathrm{m}$ ) ablöst und eine differenzierte Messung auch bei nur leichtgradig erkrankten Patienten möglich wird.

\section{Sedierung}

\section{Originalpublikationen}

Ohta Y, Miyamoto K, Kawazoe Y et al (2020) Effect of dexmedetomidine on inflammation in patients with sepsis requiring mechanical ventilation: a sub-analysis of a multicenter randomized clinical trial. Crit Care. 24:493. https://doi.org/10.1186/s13054-020-03207-8

Chen P, Jiang J, Zhang Y et al (2020) Effect of Dexmedetomidine on duration of mechanical ventilation in septic patients: a systematic review and meta-analysis. BMC Pulm Med. 20:42. https://doi.org/10.1186/s12890-0201065-6

Der antiinflammatorisch wirksame $\mathrm{a}_{2}$-Agonist Dexmedetomidin wird aufgrund seiner sedierenden, anxiolytischen und analgetischen Wirkeigenschaften zunehmend zur Sedierung von beatmeten, kritisch kranken Patienten eingesetzt und bietet möglicherweise auch einen vielversprechenden therapeutischen Ansatz. Im Umfeld operativer Eingriffe und tierexperimentell im Rahmen der Hyperinflammation konnte eine Reduktion von Entzündungsmarkern durch die Gabe von Dexmedetomidin nachgewiesen werden $[15,16]$. Der $a_{2}-\operatorname{Re}-$ zeptor auf Makrophagen scheint hierbei eine zentrale Rolle zu spielen; die Wirkung ist dosisabhängig.

Die 2017 publizierte DESIRE-Studie untersuchte ursprünglich die Auswirkung von Dexmedetomidin auf das Überleben und die Anzahl beatmungsfreier Tage, jedoch ohne signifikantes Ergebnis. Nun wurde eine bereits im Vorfeld geplante Subgruppenanalyse der 201 Patienten der DESIRE-Studie veröffentlicht [17]. Insgesamt 100 beatmete Patienten auf 8 japanischen Intensivstationen mit Sepsis erhielten Dexmedetomidin $(0,1-0,7 \mu \mathrm{g} /$ kgKG und h) zusätzlich zu Propofol und Midazolam zur Sedierung, 101 Patienten wurden lediglich mit Propofol und Midazolam sediert. Das Sedierungsziel war jeweils gleich (RASS 0 bis -2 ). Als primäre Endpunkte wurden die C-reaktives-Protein(CRP)- und Prokalzitonin(PCT)- 
Konzentrationen innerhalb der ersten 14 Tage nach Randomisierung verglichen. Es zeigte sich eine signifikante Reduktion sowohl für das CRP (Interventionsgruppe: $5,6-20,3 \mathrm{mg} / \mathrm{dl}$; Kontrollgruppe: $8,3-21,1 \mathrm{mg} / \mathrm{dl} ; \quad p=0,03$ ) als auch für das PCT (Interventionsgruppe: 1,2-37,4; Kontrollgruppe: $1,7-52,9 \mathrm{ng} / \mathrm{ml} ; p=0,04$ ). Bei den sekundären Endpunkten konnte zudem ein signifikanter Unterschied im Albuminwert zwischen den Gruppen nachgewiesen werden (Interventionsgruppe: 2,3-2,6 g/dl; Kontrollgruppe: $2,1-2,7 ; p=0,01)$. Dieser wird von den Autoren als Surrogatparameter für eine verringerte Gefäßpermeabilität unter Dexmedetomidinbehandlung interpretiert. Allerdings übertrug sich der Unterschied dieser Untersuchungsergebnisse nicht auf einen signifikanten Überlebensunterschied nach 14 Tagen. Die absolute Reduktion der Sterblichkeit von $8 \%$ (13\% vs. $21 \% ; p=0,16)$ lässt jedoch zumindest die Annahme zu, dass bei größerem Studienkollektiv auch die Verringerung der Sterblichkeit ein signifikantes Niveau erreichen könnte und fordert eine Überprüfung anhand weiterer Studien mit größerem Kollektiv. Diese Forderung unterstützt auch eine Metaanalyse, welche u.a. das Kollektiv der DESIRE-Studie einschloss und den Effekt einer Sedierung mit Dexmedetomidin auf die Beatmungsdauer untersuchte [18]. Aus den Datensätzen von 4 Studien konnte zwar kein Unterschied im primären Endpunkt, der Beatmungsdauer, gezeigt werden, jedoch ließen sich ein signifikant besseres Überleben und mehr beatmungsfreie Tage nach 28 Tagen nachweisen. All diese Hinweise auf einen positiven Effekt von Dexmedetomidin auf den Krankheitsverlauf von Patienten mit Sepsis sollten Anlass dazu geben, die Wirkung dieses Medikaments in belastbaren, prospektiven randomisierten Studien weitergehend $\mathrm{zu}$ untersuchen, um diese Erkenntnisse dann auch in die Klinik transferieren zu können.

\section{Gastrointestinale Blutung}

\section{Originalpublikation}

The HALT-IT Trial Collaborators (2020) Effects of a high-dose 24-h infusion of tranexamic acid on death and thromboembolic events in patients with acute gastrointestinal bleeding (HALT-IT): an international randomised, double-blind, placebo-controlled trial. Lancet. 395:1927-36. https://doi.org/10.1016/S01406736(20)30848-5

Die akute gastrointestinale (Gl-)Blutung ist ein lebensbedrohlicher Notfall, der abhängig von der Lokalisation (oberer oder unterer Gl-Trakt) mit einer Sterblichkeit bis zu $10 \%$ einher geht. Bei anderen Ursachen akuten und massiven Blutverlusts wie der traumatischen oder postpartalen Blutung konnte durch die Gabe von Tranexamsäure (TXA) eine Reduktion der Sterblichkeit beigleichzeitig nichterhöhtem thrombembolischen Risiko gezeigt werden [19, 20]. Auch beim Schädel-Hirn-Trauma profitieren zumindest Subgruppen von der frühzeitigen Gabe von TXA [21]. Die HALTIT-Studie untersuchte in einem von 2013 bis 2019 erhobenen Kollektiv an 164 Krankenhäusern in 15 Ländern die Auswirkung der Gabe von TXA bei Patienten mit akuter Gl-Blutung [22]. Eine Motivation für die Durchführung der Studie war u. a. eine Metaanalyse, die eine deutliche Reduktion der Sterblichkeit bei Patienten mit GI-Blutung durch die Gabe von TXA zeigte (relatives Risiko (RR): 0,61, 95\%-Konfidenzintervall (KI): 0,42-0,89) [23]. In der prospektiven randomisierten, internationalen Multizenterstudie HALT-IT erhielten 5994 Patienten zunächst $1 \mathrm{~g}$ TXA als Kurzinfusion und direkt im Anschluss $3 \mathrm{~g}$ über $24 \mathrm{~h}$, während 6015 Patienten das entsprechende Placebo ( $\mathrm{NaCl}, 0,9 \%)$ appliziert bekamen. Als Einschlusskriterien waren definiert: Patienten > 16 Jahre mit akut lebensbedrohlicher oberer oder unterer Gl-Blutung sowie die Unsicherheit des Arztes, ob eine Behandlung mit TXA durchgeführt werden soll. Hierdurch offenbart sich bereits eine Schwäche der Studie in Form eines möglicherweise relevanten Selektionsbias. So haben Patienten, die vermutlich von TXA profitieren, diese erhalten, ohne in die Studie eingeschlossen zu werden. Im primären Endpunkt, der Sterblichkeit durch ein Blutungsereignis innerhalb von 5 Tagen, war kein Unterschied zwischen den Gruppen nachzuweisen (3,7 vs. 3,8\%; RR: 0,99, $95 \%-K I: 0,82-1,18)$. Zwar war etwa die Hälfte der Todesfälle nicht auf ein Blutungsereignis zurückzuführen, doch auch die Gesamtsterblichkeit unterschied sich zwischen den Gruppen nach 28 Tagen nicht (9,5 vs. 9,2\%; RR: 1,03, $95 \%-K I: 0,92-1,16)$. Auch weitere sekundäre Endpunkte konnten keinen Behandlungsvorteil für TXA nachweisen, so unterschied sich etwa die Rate an erneuten Blutungsereignissen oder Bluttransfusionen nicht. Allerdings zeigte sich unter TXA eine erhöhte Rate von Krampfanfällen (0,6 vs. $0,4 \%$; RR: $1,73,95 \%-\mathrm{Kl}: 1,03-2,93)$ und venösen Thromboembolien (0,8 vs. 0,4\%; RR: 1,85, $95 \%-K I$ : 1,15-2,98). Somit konnten nicht nur die Ergebnisse der im Vorfeld der Studie publizierten Metaanalyse widerlegt werden; durch die erhöhte Nebenwirkungsrate muss nach der HALT-IT Studie von der routinemäßigen Gabe von TXA bei der akuten GI-Blutung abgeraten werden. Zudem zeigte sich einmal mehr, dass Metaanalysen nur der Hypothesengenerierung für große randomisierte Studien dienen sollen und die Ergebnisse nicht ohne Weiteres als Therapieempfehlung übernommen werden dürfen.

\section{Originalpublikation}

Lau JYW, Yu Y, Tang RSY et al (2020) Timing of Endoscopy for Upper Gastrointestinal Bleeding. N Engl J Med. 382:1299-308. https://doi.org/ 10.1056/NEJMoa1912484

Bei Patienten mit oberer Gl-Blutung ist häufig eine Endoskopie zur Lokalisation der Blutungsquelle und Blutstillung nötig. Empfohlen wird diese innerhalb von $24 \mathrm{~h}$ nach Aufnahme des Patienten. Ob jedoch eine noch zeitnähere Durchführung der Endoskopie einen Benefit für die Patienten bringt, ist unklar. Deshalb untersuchten Lau et al. in einer prospektiven randomisierten monozentrischen Studie in Hongkong 516 Patienten mit oberer Gl-Blutung und hohem Risiko für Tod oder weitere Blutung, definiert durch einen GlasgowBlatchford-Score über 12 [24]. Der Glasgow-Blatchford-Score kann das Risiko u.a. für Blutungsereignisse und Sterblichkeit bei Gl-Blutungen vorhersagen und reicht von 0 (niedrigstes Risiko) bis 23 (höchstes Risiko). Die Patienten erhielten entwe- 
der eine dringliche Endoskopie innerhalb von $6 \mathrm{~h}$ oder eine frühe Endoskopie zwischen 6 und $24 \mathrm{~h}$ nach gastroenterologischer Beurteilung. Als primärer Endpunkt diente die Sterblichkeit nach 30 Tagen, die sich zwischen den Gruppen nicht unterschied: dringliche Endoskopie: $8,9 \%$ vs. frühe Endoskopie: 6,6\%; (RR: 1,35, $95 \%$ $\mathrm{KI}: 0,72-2,54)$. Auch sekundäre Endpunkte konnten keine Hinweise für einen Benefit einer Endoskopie innerhalb von $6 \mathrm{~h}$ zeigen. So unterschied sich auch die Rate an Nachblutungen innerhalb von 30 Tagen nicht $(10,9 \%$ vs. $7,8 \%$; RR: $1,46,95 \%-\mathrm{Kl}$ : 0,83-2,58), ebenso war die Transfusionsrate gleich. Eine höhere Rate nachgewiesener aktiv blutender Ulzera und somit häufigeren endoskopischen Behandlungen der Blutungsquelle konnte die Nachblutungsrate oder gar die Sterblichkeit also nicht reduzieren. Möglicherweise hatte die vorausgehende antazide Therapie der Patienten in der Gruppe der Endoskopien innerhalb von $24 \mathrm{~h}$ also einen positiven Effekt auf den Verlauf der Blutung. Anhand dieser Untersuchung kann die generelle Durchführung der Endoskopie innerhalb von $6 \mathrm{~h}$ nicht empfohlen werden. Jedoch ist zu berücksichtigen, dass die Daten aufgrund des monozentrischen Studiendesigns nicht ohne Weiteres übertragbar sind und Patienten, die sich initial nicht stabilisieren ließen und so eine dringliche Behandlung erfahren hatten, nicht eingeschlossen wurden.

\section{Korrespondenzadresse}

\section{Prof. Dr. med. M. A. Weigand}

Klinik für Anästhesiologie, Universitätsklinikum Heidelberg

Heidelberg, Deutschland

markus.weigand@med.uni-heidelberg.de

Interessenkonflikt. M. Dietrich, C. Beynon, M. O. Fiedler, M. Bernhard, P. Kümpers, A. Hecker, C. Jungk, C. Nusshag, D. Michalski, T. Brenner, M. A. Weigand und C. J. Reuß geben an, dass kein Interessenkonflikt besteht.

\section{Literatur}

1. Johns Hopkins University (2021) COVID-19 dashboard by the Center for Systems Science and Engineering (CSSE) at Johns Hopkins University (JHU). https://coronavirus.jhu.edu/map.html. Zugegriffen: 26. März 2021

2. Marik PE et al (2017) Hydrocortisone, vitamin C, and thiamine for the treatment of severe sepsis and septic shock: a retrospective before-after study. Chest 151(6):1229-1238

3. Fowler AA 3rd et al (2019) Effect of vitamin $C$ infusion on organ failure and biomarkers of inflammation and vascular injury in patients with sepsis and severe acute respiratory failure: the CITRIS-ALI randomized clinical trial. JAMA 322(13):1261-1270

4. Fujii T et al (2020) Effect of vitamin C, hydrocortisone, and thiamine vs hydrocortisone alone on time alive and free of vasopressor support among patients with septic shock: the VITAMINS randomized clinical trial. JAMA 323(5):423-431

5. Colliou E et al (2017) Oxalate nephropathy following vitamin $\mathrm{C}$ intake within intensive care unit. Clin Nephrol 88(12):354-358

6. McHugh GJ, Graber ML, Freebairn RC (2008) Fata vitamin $\mathrm{C}$-associated acute renal failure. Anaesth Intensive Care 36(4):585-588

7. Moskowitz A et al (2020) Effect of ascorbic acid corticosteroids, and thiamine on organ injury in septic shock: the ACTS randomized clinical trial. JAMA 324(7):642-650

8. Hwang SY et al (2020) Combination therapy of vitamin $\mathrm{C}$ and thiamine for septic shock: a multicentre, double-blinded randomized, controlled study. Intensive Care Med 46(11):2015-2025

9. Iglesias J et al (2020) Outcomes of metabolic resuscitation using ascorbic acid, thiamine, and glucocorticoids in the early treatment of sepsis: the ORANGES trial. Chest 158(1):164-173

10. Sevransky JE et al (2021) Effect of vitamin C, thiamine, and hydrocortisone on ventilator- and vasopressor-free days in patients with sepsis: the VICTAS randomized clinical trial. JAMA 325(8):742-750

11. Hasegawa D et al (2021) Effect of ultrashortacting beta-blockers on mortality in patients with persistent tachycardia despite initial resuscitation: a systematic review and meta-analysis of randomized controlled trials. Chest 159(6):2289-2230

12. Singer $M$ et al (2016) The third international consensus definitions for sepsis and septic shock (sepsis-3). JAMA 315(8):801-810

13. SepNet Critical Care Trials Group (2016) Incidence of severe sepsis and septic shock in German intensive care units: the prospective, multicentre INSEP study. Intensive Care Med 42(12):1980-1989

14. Brechot $\mathrm{N}$ et al (2020) Venoarterial extracorporeal membrane oxygenation to rescue sepsisinduced cardiogenic shock: a retrospective, multicentre, international cohort study. Lancet 396(10250):545-552

15. Taniguchi Tet al (2004) Effects of dexmedetomidine on mortality rate and inflammatory responses to endotoxin-induced shock in rats. Crit Care Med 32(6):1322-1326

16. Li $Y$ et al (2015) Effect of dexmedetomidine on early postoperative cognitive dysfunction and peri-operative inflammation in elderly patients undergoing laparoscopic cholecystectomy. Exp Ther Med 10(5):1635-1642

17. Ohta $Y$ et al (2020) Effect of dexmedetomidine on inflammation in patients with sepsis requiring mechanical ventilation: a sub-analysis of a multicenter randomized clinical trial. Crit Care 24(1):493

18. Chen P et al (2020) Effect of dexmedetomidine on duration of mechanical ventilation in septic patients: a systematic review and meta-analysis. BMC Pulm Med 20(1):42

19. WOMAN Trial Collaborators (2017) Effect of early tranexamic acid administration on mortality, hysterectomy, and other morbidities in women with post-partum haemorrhage (WOMAN): an international, randomised, double-blind, placebocontrolled trial. Lancet 389(10084):2105-2116

20. CRASH-2 Trial Collaborators, et al. (2010) Effects of tranexamic acid on death, vascular occlusive events, and blood transfusion in trauma patients with significant haemorrhage (CRASH-2): a randomised, placebo-controlled trial. Lancet 376(9734):23-32

21. CRASH-3 Trial Collaborators (2019) Effects of tranexamic acid on death, disability, vascular occlusive events and other morbidities in patients with acute traumatic brain injury (CRASH-3): a randomised, placebo-controlled trial. Lancet 394(10210):1713-1723

22. HALT-IT Trial Collaborators (2020) Effects of a highdose 24-h infusion of tranexamic acid on death and thromboembolic events in patients with acute gastrointestinal bleeding (HALT-IT): an international randomised, double-blind, placebocontrolled trial. Lancet 395(10241):1927-1936

23. Gluud LL, Klingenberg SL, Langholz E (2012) Tranexamic acid for upper gastrointestinal bleeding. Cochrane Database Syst Rev 1:CD6640

24. Lau JYW, Yu Y, Chan FKL (2020) Timing of endoscopy for acute upper gastrointestinal bleeding. Reply. N Engl J Med 383(4):e19 